\title{
Mesial Temporal Lobe Epilepsy Lateralization Using SPHARM-Based Features of Hippocampus and SVM
}

\author{
Mohammad Esmaeilzadeh $^{* a, b}$, Hamid Soltanian-Zadeh ${ }^{* b, c}$, Kourosh Jafari-Khouzani ${ }^{{ }^{*} \mathrm{c}}$ \\ ${ }^{a}$ Research School of Information Science and Engineering, Australian National University, \\ Canberra, ACT 0200, Australia \\ ${ }^{\mathrm{b}}$ Control and Intelligent Processing Centre of Excellence, School of Electrical and Computer \\ Engineering, Faculty of Engineering, University of Tehran, Tehran 14395-515, Iran \\ ${ }^{\mathrm{c}}$ Dept. of Diagnostic Radiology, Henry Ford Hospital, Detroit, MI 48202 USA
}

\begin{abstract}
This paper improves the Lateralization (identification of the epileptogenic hippocampus) accuracy in Mesial Temporal Lobe Epilepsy (mTLE). In patients with this kind of epilepsy, usually one of the brain's hippocampi is the focus of the epileptic seizures, and resection of the seizure focus is the ultimate treatment to control or reduce the seizures. Moreover, the epileptogenic hippocampus is prone to shrinkage and deformation; therefore, shape analysis of the hippocampus is advantageous in the preoperative assessment for the Lateralization. The method utilized for shape analysis is the Spherical Harmonics (SPHARM). In this method, the shape of interest is decomposed using a set of bases functions and the obtained coefficients of expansion are the features describing the shape. To perform shape comparison and analysis, some pre- and post-processing steps such as "alignment of different subjects' hippocampi" and the "reduction of featurespace dimension" are required. To this end, first order ellipsoid is used for alignment. For dimension reduction, we propose to keep only the SPHARM coefficients with maximum conformity to the hippocampus shape. Then, using these coefficients of normal and epileptic subjects along with $3 D$ invariants, specific lateralization indices are proposed. Consequently, the 1536 SPHARM coefficients of each subject are summarized into 3 indices, where for each index the negative (positive) value shows that the left (right) hippocampus is deformed (diseased). Employing these indices, the best achieved lateralization accuracy for clustering and classification algorithms are $85 \%$ and $92 \%$, respectively. This is a significant improvement compared to the conventional volumetric method.
\end{abstract}

Keywords: Hippocampus Shape Analysis, Mesial Temporal Lobe Epilepsy (mTLE), mTLE Lateralization, Spherical Harmonics (SPHARM), 3D Representation and Registration, Support Vector Machine (SVM).

\section{INTRODUCTION}

In recent years, shape analysis of brain structures has achieved great importance in the context of medical image computing. The increased research in this field has been inspired by the importance of neurodegenerative disorders (such as Alzheimer's, Schizophrenia, Parkinson and Epilepsy) and also evidences that show relations between these diseases and deformation of the brain structures [1-3].

\subsection{Hippocampus and Mesial Temporal Lobe Epilepsy}

Hippocampus (HC) is a brain structure that belongs to the limbic system and is located in the mesial temporal lobe of the brain. It plays important roles in the short-term memory, the formation of memories and language tasks [4]. Hence, HC is a major structure of interest in many researches relevant to the above mentioned disorders.

Temporal lobe epilepsy (TLE) is one of the most important types of focal epilepsy where the origination of the epileptic seizures is from the temporal lobe of the brain. More specifically, when the focus of the seizures is in the middle part of the temporal lobe (e.g. hippocampus), it is called mesial temporal lobe epilepsy (mTLE). For the patients with refractory mTLE suffering from intractable seizures, hippocampal resection is the ultimate treatment. For this treatment to be most

mohammad.esmaeilzadeh@anu.edu.au,hszadeh@ut.ac.ir, \{hamids, kjafari\}@rad.hfh.edu

Medical Imaging 2012: Image Processing, edited by David R. Haynor, Sébastien Ourselin,

Proc. of SPIE Vol. 8314, 83144H - @ 2011 SPIE - CCC code: 0277-786X/11/\$18 · doi: 10.1117/12.911740

Proc. of SPIE Vol. $831483144 \mathrm{H}-1$ 
effective in controlling the seizures (with minimal side-effects), accurate identification of the epileptogenic HC (Lateralization) is critical.

In mTLE, the most frequent pathologic finding is hippocampal sclerosis, which is the neuronal cell loss caused by seizures [5]. Considering the evidences of $\mathrm{HC}$ deformation due to hippocampal sclerosis [3, 5, 6], hippocampal volumetry is the conventional method for lateralization. However, these deformations do not always alter the volume, but sometime just the shape. So shape analysis can be beneficial in providing additional morphometric features and improving the lateralization accuracy.

\subsection{Shape Analysis Methods}

As mentioned above, hippocampal volumetry along with some other MRI measures are the conventional methods for studying hippocampi variations. Although volumetry is capable of revealing major deformations and global differences in some disorders [7], it is blind to minuscule shape changes. So, in order to better compare the HCs, researches have focused on quantitative shape analysis methods with better discriminant features. Some researchers have proposed to apply deformable registration to a template $[1,8,9]$. Despite problems of template selection and high dimensionality of transformation, these studies achieved reasonable results. The methods in $[10,11]$ were among the first methods proposed for 3D shape analysis based on sampled descriptions. Cootes et al. [12] proposed Point Distribution Model for 3D shape analysis and deformation study. Other shape analysis methods based on medial shape descriptions in 3D and 2D were proposed by Styner [13] and Golland [14], respectively. Besides these, some methods build a simplified representation of anatomical structure by utilizing shape descriptors, such as spherical harmonics (SPHARM) [15], spherical wavelets [16], and Laplace-Beltrami operator [17]. In these methods, shape (volume) or surface is decomposed into series of bases and the coefficients are used as descriptive features.

SPHARM, a 3D extension of Fourier analysis, is what we utilize in this paper. Applications of SPHARM have been widely reported in many articles. Styner et al. [18] developed a framework using SPHARM to analyze caudate and hippocampus shape in schizophrenia. Zhao et al. [19] used the same framework to analyze hippocampus shape in latelife depression. McKeown et al. [20] employed SPHARM for thalami shape analysis in Parkinson's disease (PD). They found some differences between "control and PD" thalami and also "left and right" thalami in each group that volumetric analysis was unable to distinguish between them. SPHARM has been widely used for hippocampus shape analysis in Alzheimer's disease (AD) [21-23]. Gerardin et al. [24] obtained 88\% accuracy in distinguishing AD from normal aging by hippocampus shape analysis using SPHARM.

In epilepsy, shape analysis has not been as extensively researched compared to Alzheimer's and Schizophrenia. Some of the researches have focused on statistical analysis of the hippocampus shape [25, 26]. These works provide a localized 3D probabilistic surface that reveals the extent by which the HC shape is deformed in comparison with control subjects. In some other works, like those by Vemuri and his research group [27, 28], hippocampus shape analysis has been used for distinguishing between controls and epileptic patients, and also for lateralization. Recently, [29] has used the same dataset that we utilize in this research, and they distinguished between healthy and control subjects by hippocampus shape analysis using Laplace-Beltrami spectrum.

\subsection{Our Contribution}

We build upon our previous work [30] and perform SPHARM-based shape analysis of HCs. We utilize SPHARM coefficients and benefit from SVM classifiers to improve mTLE lateralization. The rest of this paper is organized as follows. In Section 2, we first introduce the datasets. Then we describe SPHARM-based shape representation and our proposed method for SPHARM registration. Next, we describe our feature selection and extraction methods. In Section 3 , we provide the lateralization results, obtained using clustering of SPHARM coefficients and volumetry of the HCs. In addition, we present the achieved results using SVM classification. Finally, we conclude in Section 4 with discussion and future works.

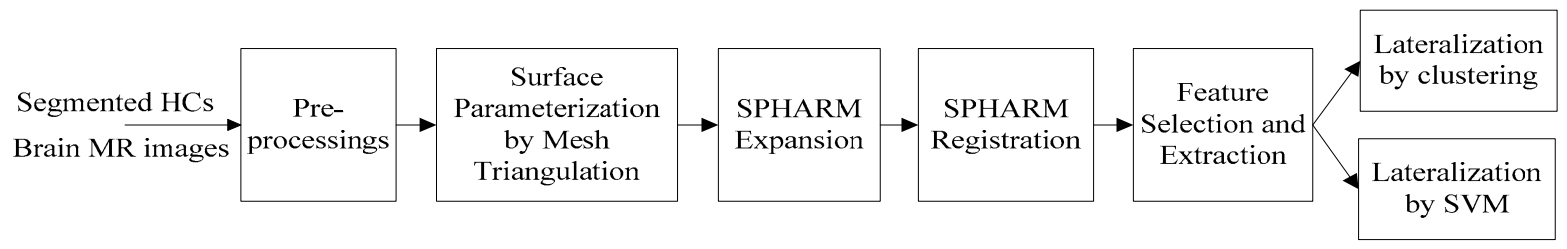

Figure 1. The proposed procedure for shape analysis of hippocampus using SPHARM. 


\section{MATERIAL AND METHODS}

\subsection{Data}

We use T1-weighted (T1W) MR provided by Department of Diagnostic Radiology at Henry Ford Hospital (HFH). This dataset contains T1W images of 25 healthy ("N"; 13 males, 12 females; age range 19-54, mean \pm SD = $32 \pm 9$ ) and 66 epileptic ("EP"; 24 males, 42 females; age range 15-66, mean $\pm \mathrm{SD}=40 \pm 11$ ) subjects. Images are either $256 \times 256$, voxel size $=0.78 \times 0.78 \times 2 \mathrm{~mm}^{3}$ or $512 \times 512$, voxel size $=0.39 \times 0.39 \times 2 \mathrm{~mm}^{3}$ (Slice thickness $=2 \mathrm{~mm}$ ). Therefore, images should undergo the same structural standardization described in [30].

For all images, accurate segmentation of HCs was made available, by manually outlining hippocampal contours with reference to an MRI atlas identifying the Hippocampus [4]. The segmentation technique is described comprehensively in [31], and the resultant dataset is available online ${ }^{1}$.

To detect the epileptogenic HCs, all patients underwent a full complement of preoperative studies such as videoelectroencephalographic (vEEG) inpatient assessment over a minimum three-day period, MR imaging, sodium amobarbital study, and neuropsychological assessment. Some of the patients required extraoperative electrocorticography (ECoG) with intracranially implanted electrode arrays. The outcome revealed that in 33 of the patients $(50 \%)$, the right HCs were epileptogenic and in the other $33(50 \%)$ the left ones were epileptogenic. These finding were confirmed by seizure freedom after surgical removal of the presumed epileptogenic hippocampus and clinical follow-ups.

\subsection{Pre-Processing and HCs Surface Parameterization}

As described in [30], since not all images are with similar voxel size, mapping to Montreal Neurological Institute (MNI) atlas is performed to generate a common framework for all images. Additionally, the mirroring of the right hippocampi omits the directional differences of left (L) and right (R) HCs and makes the shape comparison of L and R HCs feasible. An Instance of segmented HCs mapped on MNI template is shown in Figure 2.
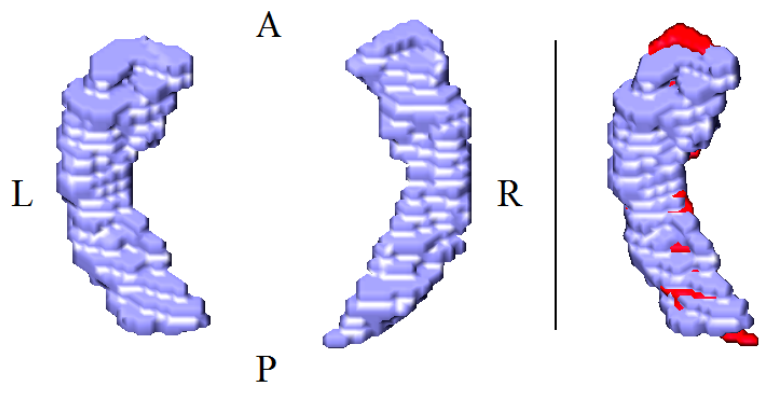

Figure 2. Segmented EP-HCs mapped on the MNI template; (left) Top view of both hippocampi of a subject, (right) mirrored right $\mathrm{HC}$ [red] on the left one [blue]. (In grayscale printing, red appears darker than blue)

Finally, before the segmented HC are being fed into SPHARM expansion, their surface should be parameterized. This is achieved by use of triangular meshes and is described in [30].

\subsection{SPHARM}

SPHARM was proposed by Brechbühler et al. [15] for modeling arbitrarily shaped but simply connected 3D objects. SPHARM, similar to volume, is a global shape analysis framework, but its multi-scale characteristics make it superior over simple volumetric analysis. As shown in Fig. 1, often three steps are taken in a typical SPHARM analysis:

(1) Surface parameterization

(2) SPHARM expansion

(3) SPHARM registration

\footnotetext{
${ }^{1}$ http://www.radiologyresearch.org/HippocampusSegmentationDatabase/
} 
We briefly addressed the surface parameterization in 2.2. In this section, we focus on SPHARM expansion and we will discuss SPHARM registration in 2.4. The mathematics behind SPHARM has been fully described in [15] and also in [23]. Here we provide just the fundamentals required to describe our methods.

SPHARM basis functions of degree $l$ and order $m\left(Y_{l}^{m},-l \leq m \leq l\right)$ are defined as follows:

$$
\begin{aligned}
Y_{l}^{m}(\theta, \phi) & =\sqrt{\frac{2 l+1(l-m) !}{4 \pi(l+m) !}} P_{l}^{m}(\cos \theta) e^{i m \phi} \\
& \theta \in[0: \pi], \phi \in[0: 2 \pi)
\end{aligned}
$$

where $P_{l}^{m}(\cos \theta)$ are the associated Legendre polynomials defined by the differential equation:

$$
P_{l}^{m}(w)=\frac{(-1)^{m}}{2^{l} l !}\left(1-w^{2}\right)^{m / 2} \frac{d^{m+l}}{d w^{m+l}}\left(1-w^{2}\right)^{l}
$$

Therefore, considering a parameterized surface $\vec{v}(\theta, \phi)$ in spherical coordinate, the SPHARM expansion takes the form:

$$
\begin{aligned}
& \vec{v}(\theta, \phi)=(x(\theta, \phi), y(\theta, \phi), z(\theta, \phi))^{T}=\sum_{l=0}^{\infty} \sum_{m=-l}^{l} \vec{c}_{l}^{m} Y_{l}^{m}(\theta, \phi) \\
& \vec{c}_{l}^{m}=\left(c_{l x}^{m}, c_{l y}^{m}, c_{l z}^{m}\right)^{T}
\end{aligned}
$$

The coefficients $\vec{c}_{l}^{m}$ up to a user-desired degree $\left(L_{\max }\right)$ can be estimated by solving a set of linear equations in a least squares fashion. The object surface can be reconstructed by these coefficients and using more coefficients leads to a more detailed reconstruction [21]. We set $L_{\max }=15$ to keep appropriate amount of details of HC shape. This results in $\left(L_{\max }+1\right)^{2}=256 \vec{c}_{l}^{m}$ coefficients for each HC. Considering $x, y$, and $z$ elements of $\vec{c}_{l}^{m}$, the total number of features for each $\mathrm{HC}$ will be $256 \times 3=768$. These 768 features contain information about HCs' shape as well as position and orientation. So, before comparing various HCs' shape by analyzing these 768 features, they need to be aligned. To this end, methods to deal with the alignment (registration) of various HCs in the feature space will be described in the following section. Figure 3 depicts some parameterized HCs of healthy and epileptic subjects and also the reconstructed HCs when $L_{\max }=15$.

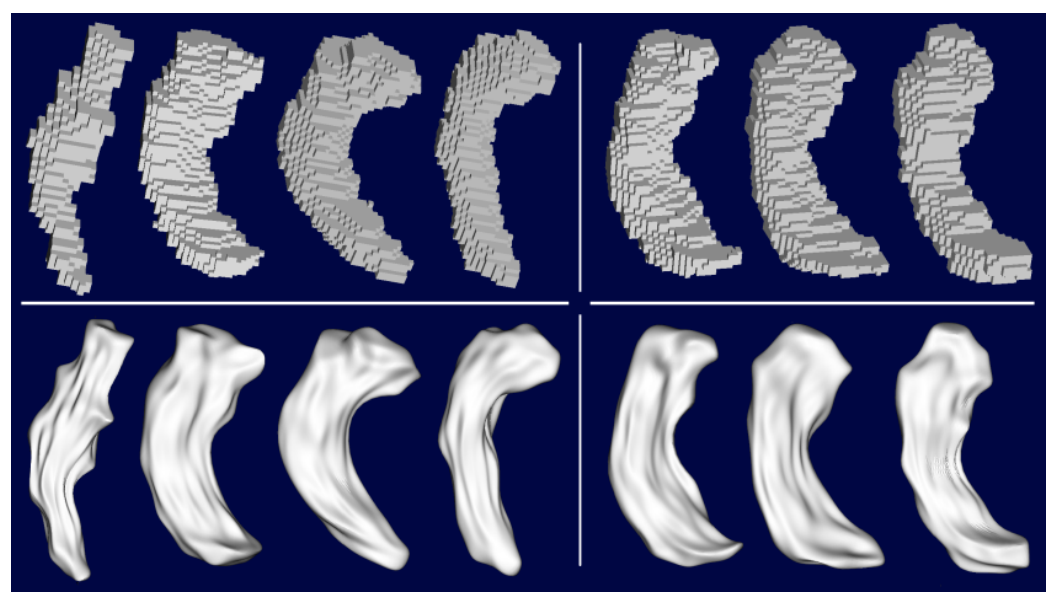

Figure 3. Some samples of (up) parameterized HCs, and (down) reconstructed HCs with $L_{\max }=15$; (left) epileptic HCs, (right) normal HCs.

\subsection{Registration}

The whole brain registration introduced as a pre-processing in section 2.2 can slightly help with bringing the HCs to a common framework. However, due to the variable $\mathrm{HC}$ position and orientation in different subjects, fine tuning is still of great importance. In other words, without SPHARM registration, the coefficients are not comparable. 
To cope with this problem, some methods have been proposed in the literature. Mckeown et al. [20] uses the 3D invariants proposed in [32]. In this method, SPHARM coefficients are considered as tensors. By applying the concepts of tensor theory, any dependency upon orientation is eliminated. Shen et al. [22] uses landmarks for registration of SPHARM objects. In his method, landmarks are manually placed on some corresponding surface points of all subjects. By minimizing the root mean square distance between landmark vectors of two subjects, they are aligned. This step is not performed in the object space; rather it is performed in the SPHARM feature space.

Both of the above methods are efficient but very complicated. The latter could also be greatly subjective. Here we describe our method for SPHARM registration, which is conducted in the SPHARM feature space.

Talking about registration, usually scaling, translation and rotation are required. In our problem, scaling is not an issue since various brains have been already scaled to be mapped on the MNI atlas.

About the translation, as described in [18], we can easily normalize the position of HCs by setting $x, y$, and $z$ elements of $\vec{c}_{0}^{0}$ to zeros. This brings the center of all HCs into Cartesian origin of $(0,0,0)$ and reduces the number of features to 765 .

For the rotation, we propose a simple yet practical method by benefiting from the SPHARM description of degree 1, which is called first order ellipsoid (FOE). The following steps can briefly express this method:

1- Compute the FOE of each HC.

2- Represent it using [vertices, faces] format described in [30] for parameterization by triangular meshes.

3- Calculate principal components (PCs) of the matrix 'vertices' (3 principal components exist).

4- Compute the $3 \times 3$ matrix $\mathrm{M}$ for mapping these three principal components on the three main axes.

5- Multiply SPHARM coefficients by M to obtain new coefficients $\vec{c}_{l}^{\prime m}$.

Since $\vec{c}_{l}^{m}$ consists of $x, y$, and $z$ elements, applying the same mapping on the corresponding coefficients is possible. Modified coefficients generated by this method are representative of shapes with complete alignment on the Cartesian axes, so $\vec{c}_{l}^{\prime m}$ will be used to compare different hippocampi, such as $\mathrm{N}$ and EP ones. Figure 4 depicts an example of mapping PCs on the main axes and also a sample result of our method for fine alignment.

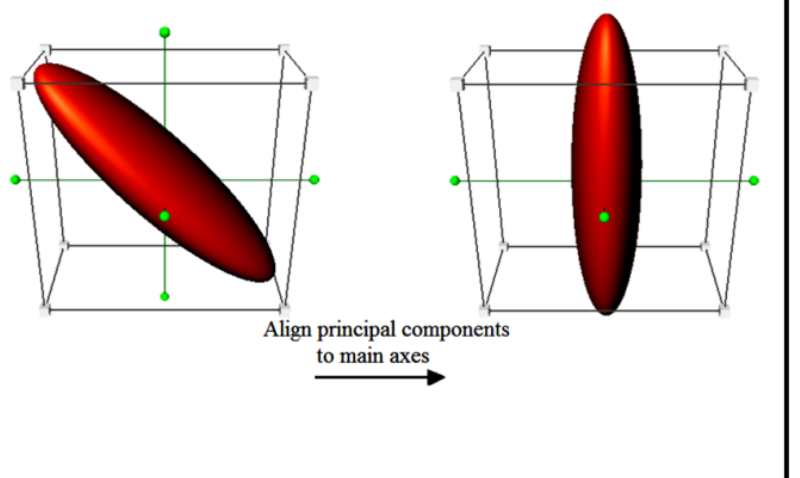

(a)

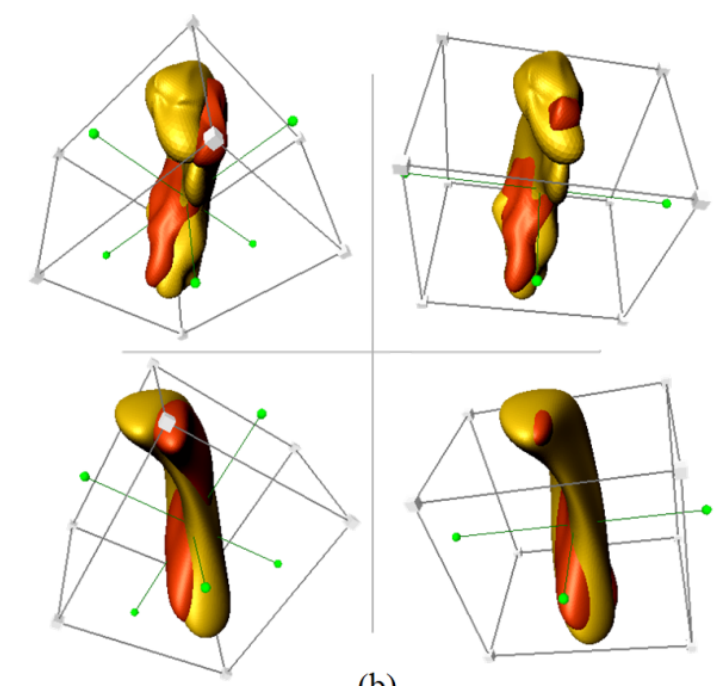

(b)

Figure 4. (a) Mapping PCs of FOE to main axes for SPHARM registration in feature space. (b) SPHARM description of two subjects' HC; (left) Raw SPHARM description, (right) Aligned SPHARM description by mapping PCs of FOE on main Cartesian axes, (top) Degree 15 SPHARM description, (bottom) Degree 5 SPHARM description.

\subsection{Feature Selection for Lateralization}

The final step is to extract meaningful features from SPHARM coefficients in order to lateralize the mTLE patients. In [30], we have proposed three Lateralization Indices (LIs) based on SPHARM, and also one standard LI based on Volumetry. These indices are brought in Table 1; we briefly describe them here. 
Table 1. Definition of three SPHARM-based LIs and the LI based on volume.

\begin{tabular}{|c|c|c|c|}
\hline Vol & ee I & ects & 3. Self-d \\
\hline$L I_{v o l}=\frac{L_{v o l}-R_{v o l}}{L_{v o l}+R_{v o l}}$ & $L I_{S D 1}=\sum_{i=1}^{20} \frac{|C L(i)|-|C R(i)|}{|C L(i)|+|C R(i)|}$ & $\begin{array}{c}L I_{D 2 N}=\sum_{j=1}^{2 N} \operatorname{sign}\left(D I S T_{R}(j)-D I S T_{L}(j)\right) \\
D I S T_{X}(j)=\sum_{k=1}^{20}\left(C X(k)-C N_{j}(k)\right)^{2}, X=L \text { or } R\end{array}$ & $\begin{array}{c}L I_{S D 2}=\sum_{l=1}^{L_{\max }} \frac{|N L(i)|-|N R(i)|}{|N L(i)|+|N R(i)|} \\
N X(l)=\sum_{m=-l}^{l}\left|\vec{c}_{l}^{\prime m}\right|\end{array}$ \\
\hline
\end{tabular}

$L I_{v o l}$ is the simplest index where $L_{v o l}$ and $R_{v o l}$ are volumes of the L and R HCs. Negative (positive) values of $L I_{v o l}$ indicate smaller left (right) hippocampus. In mTLE, the smaller hippocampus is more likely to be epileptogenic. For normal subjects, this index is usually around zero.

As detailed in the previous sections, each HC after SPHARM registration is described by 765 coefficients, which are positive and negative real numbers. Since not all of them are useful, some coefficients selection is necessary. We select the coefficients that have the same sign (+ or - ) for all $\mathrm{L}$ and $\mathrm{R}$ hippocampi of normal and epileptic subjects. This leads to 20 coefficients (CL and CR) for each HC. This method of selection is justified by the fact that the coefficients with multiple changes in sign for different subjects are not accurate representer of $\mathrm{HC}$ shape, as they are not matched sufficiently to the $\mathrm{HC}$ shape and thus are not consistent.

Using $C L$ and $C R$ of patients and $C N$ (Normal subjects' $C L$ and $C R$ ), $L I_{S D I}$ and $L I_{D 2 N}$ are obtained. $L I_{S D I}$ definition is exactly similar to $L I_{v o l}$ but in a multi-scale manner. $L I_{D 2 N}$ is defined in a way to reflect the degree in which each $\mathrm{HC}$ is similar to Normal HCs. $2 \mathrm{~N}$ is the total number of normal left and right HCs.

Another method to establish a criterion for measuring left-right HCs distance is using the simplest invariants discussed in [32] that results to $L I_{S D 2}$. This is a multi-scale method and summarizes all the coefficients to $L_{\max }+l$ values called $N(l)$. These $L_{\max }+1$ values are used to generates another lateralization index based on self-distance.

In section 3, we benefit from these indices and provide the results of simple clustering and classification by SVM for mTLE lateralization.

\section{RESULTS}

\subsection{Simple Clustering by Volumetric Analysis}

By simple clustering we mean deciding on each patient just by using the sign of its own LIs, with no training. So by using $L I_{v o l}$ and simple clustering (if $L I_{v o l}<0, \mathrm{~L}-\mathrm{HC}$ is epileptogenic and if $L I_{v o l}>0, \mathrm{R}-\mathrm{HC}$ is epileptogenic), lateralization accuracy of $78.8 \%$ is achieved (52 out of 66). However, as table 2 suggests, there exists an overlap between the range of $L I_{v o l}$ for EP and N subjects.

Table 2. $L I_{v o l}$ for healthy and epileptic subjects, STD=Standard Deviation.

\begin{tabular}{lccccc}
\hline & No. Of subjects & Mean $L I_{\text {vol }}$ & STD $L I_{\text {vol }}$ & Max $L I_{\text {vol }}$ & Min $L I_{\text {vol }}$ \\
\hline Healthy & 25 & -0.002 & 0.037 & 0.079 & -0.071 \\
\hline Epileptic & 66 & -0.033 & 0.189 & 0.349 & -0.539 \\
\hline
\end{tabular}

So, we divide EPs into two groups by considering the range of $L I_{v o l}$ for $\mathrm{N}$ subjects as $[\mu-2 \sigma, \mu+2 \sigma] \approx[-0.08,0.08]$ :

- 40 Patients with $L I_{v o l}$ outside the range for normal subject (the $L I_{v o l}$-based lateralization accuracy was $92.5 \%$ ),

- 26 Patients with $L I_{v o l}$ inside the range for normal subject (the $L I_{v o l}$-based lateralization accuracy was 57.7\%).

The former can be confidently classified as R or L, but the latter will remain as the input to the next analysis steps, which are SPHARM analysis.

\subsection{Simple Clustering by SPHARM Analysis}

Here, we calculate the three SPHARM-based LIs, defined in Table 1, for all normal and epileptic subjects. Our method failed in the calculation of the SPHARM coefficients for one subject ${ }^{1}$. Hence, 25 EP subjects of HFH with $\left|L I_{\text {vol }}\right| \leq 0.08$

\footnotetext{
${ }^{1}$ This subject was lateralized correctly by volumetric clustering
} 
are considered in this level of analysis. Again, we find the confidence interval of normal state for each of these features, and classify those subjects outside the bounds. Table 3 gives some information about these LIs.

Table 3. Mean, standard deviation, maximum, and minimum value for SPHARM-based LIs. N="25 normal subjects", EP="25 epileptic subjects with $\left|L I_{\text {vol }}\right| \leq 0.08$ ".

\begin{tabular}{llllll}
\hline & & Mean & STD & Max & Min \\
\hline \multirow{2}{*}{$L I_{S D 1}$} & $\mathrm{~N}$ & 0.28 & 4.87 & 7.94 & -11.74 \\
\cline { 2 - 6 } & $\mathrm{EP}$ & 1.15 & 8.57 & 14.96 & -19.66 \\
\hline \multirow{2}{*}{$L I_{S D 2}$} & $\mathrm{~N}$ & 0.04 & 1.14 & 2.32 & -1.78 \\
\cline { 2 - 6 } & $\mathrm{EP}$ & 0.32 & 2.12 & 4.52 & -3.13 \\
\hline \multirow{2}{*}{$L I_{D 2 N}$} & $\mathrm{~N}$ & 0.05 & 1.10 & 3.00 & -2.00 \\
\cline { 2 - 6 } & $\mathrm{EP}$ & -0.4 & 2.89 & 6.00 & -7.00 \\
\hline
\end{tabular}

Almost in all epileptic cases (except three of them), three LI values have the same sign, and all subjects have at least one LI outside the normal state intervals. So, the simple clustering is performed and the results are provided in Table 4.

Table 4. Results of simple clustering for EP HCs lateralization, $\mathrm{CL}=\mathrm{Correctly}$ Lateralized.

\begin{tabular}{lccc}
\hline & No. of subjects & No. of CL, VOL & No. of CL, SPHARM \\
\hline$\left|L I_{\text {vol }}\right| \leq 0.08$ & $26(25$ in SPHARM) & $15(57.7 \%)$ & $18(72.0 \%)$ \\
\hline$\left|L I_{v o l}\right|>0.08$ & 40 & $37(92.5 \%)$ & $37(92.5 \%)$ \\
\hline EP - total & $66(65$ in SPHARM) & $52(78.8 \%)$ & $55(84.6 \%)$ \\
\hline
\end{tabular}

Considering the above results and the footnote in previous page, it is evident that in patients with $\left|L I_{v o l}\right| \leq 0.08$, using SPHARM has increased the No. of CL by 4 . It is also clearly shown that the SPHARM method produced exactly the same result for those with $\left|L I_{\text {vol }}\right|>0.08$, of course not only in overall CL ratio, but also in subject wise analysis.

\subsection{Classification of Patients Using SVM}

Up to this level of our analysis for mTLE lateralization, a simple clustering of the patients was performed; that is we only relied on normal state of HCs and deviations from this state was used to find the diseased HCs. In other word, our diagnostic system was trained using the normal subjects and the only prior knowledge considered regarding the epileptic HCs was that they may get deformed due to the disease. In addition, LIs were interpreted independently.

Therefore, in order to improve the sensitivity of our system, first we consider LIs jointly. Then, we train our system with the features of the diseased hippocampi. This procedure is done for the 25 epileptic patients with $\left|L I_{v o l}\right| \leq 0.08$, because the other 40 showed reasonable results in the previous steps.

Through a close inspection of the LIs features in $3 \mathrm{D}$, we noticed that $L I_{S D 2}$ and $L I_{D 2 N}$ can together separate the patients with the left seizure foci from those with the right seizure foci. This is shown in Figure 5.

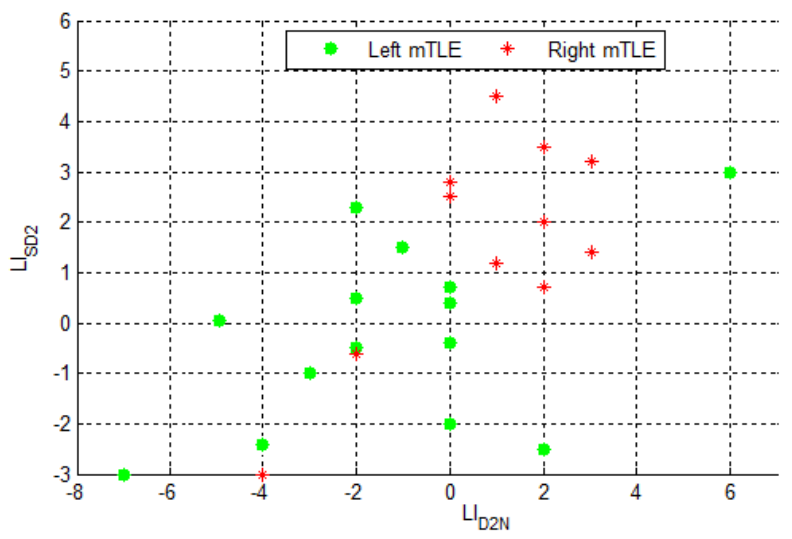

Figure 5. 2D feature space of $L I_{S D 2}$ and $L I_{D 2 N}$. 
Therefore, we utilized these two features along with a Support Vector Machine (SVM) classifier to test their classification ability. The arrangements of the data points in 2D feature spaces suggest using linear SVM and SVM with Radial Basis Function (RBF). 20 subjects are used in the training phase while the remaining 5 subjects are used in the testing phase. This is repeated for various combinations of [20 train, 5 test subjects] and the average of the correctly classified ratios (ACCR) is calculated which turns out to be $89 \%$ for the SVM-RBF and 74\% for the linear SVM (5-fold cross-validation).

To test the generalization power of our system, we leave $\mathrm{K}$ samples out (various values of $\mathrm{K}$ ) and evaluate the performance of the system [33]; results are provided in Table 5.

Table 5. K samples out cross validation. Classification accuracy of the second group of patients (25 EPs), using linear SVM and SVM with Radial Basis Function (RBF).

\begin{tabular}{cccccc}
\hline & $\mathrm{K}=1$ (leave one out) & $\mathrm{K}=2$ & $\mathrm{~K}=5$ (5-fold cross validation) & $\mathrm{K}=7$ & $\mathrm{~K}=10$ \\
\hline SVM-RBF & $\mathbf{9 2 \%}$ & $91.2 \%$ & $89.1 \%$ & $87.5 \%$ & $83.6 \%$ \\
\hline Linear SVM & $80 \%$ & $75.5 \%$ & $73.9 \%$ & $70.5 \%$ & $67.1 \%$ \\
\hline
\end{tabular}

Note that the SVM-RBF has generated satisfactory results for the classification of the left and right mTLE patients. Figure 6 depicts examples of Linear and RBF SVM.
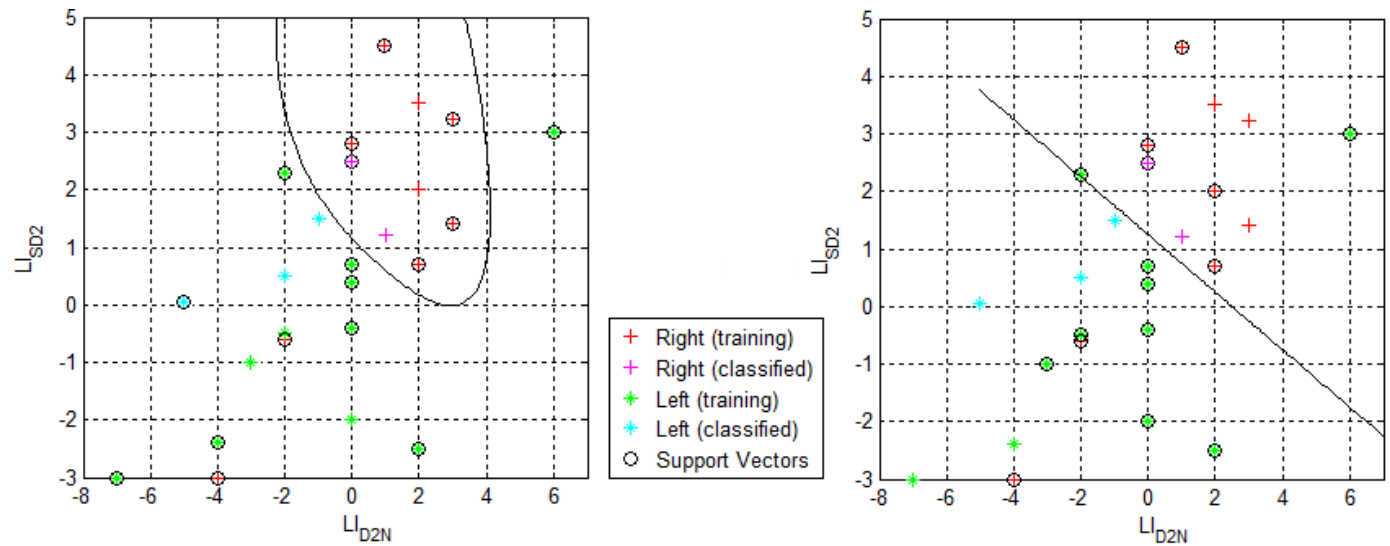

Figure 6. Example results of 5-fold cross-validation for SVM classification of left and right mTLE. (left) SVMRBF (right) linear SVM.

\section{CONCLUSION AND DISCUSSION}

In this article, we made use of a well-known shape analysis framework, SPHARM, for lateralization in mTLE.

To perform a comparison between healthy and epileptic subjects, some pre-processing such as voxel-size unification and rigid registration of the brain images are performed. Next, SPHARM-based representations of the HCs are achieved by calculating the coefficients. Then, we use our simple method for fine alignment of the HCs. The method is based on mapping the principal components of the FOE of each $\mathrm{HC}$ on Cartesian axes, and is carried out in the space of SPHARM coefficients. Finally, some features are extracted and the corresponding LIs are calculated.

The utilization of the extracted features for lateralization is carried out in two distinct manners: clustering and classification. The former uses the normal hippocampi to train the system, but in the latter, epileptic hippocampi are involved in the training phase.

About the clustering, as Table 4 suggests, the SPHARM-based method has improved the lateralization accuracy for patients with $\left|L I_{v o l}\right| \leq 0.08$. This improvement is achieved by the extra information in SPHARM coefficients in comparison to volume. If volume of a shape is considered to be equivalent to energy of a signal, then SPHARM coefficients will be the same as the Fourier coefficients; obviously the latter provide more information about our signal (shape) of interest. Also, similar to 1D Fourier expansion, in 3D analysis, the problem of choosing an optimum value for $L_{\max }$ (similar to maximum frequency in 1D signals) to keep appropriate amount of details while preventing noise, exists. Hence, the trade-off among dimensionality, accuracy, and sensitivity to noise should be considered. 
The above discussion is also applicable to support the method of feature selection for $C L$ and $C R$. In 1D signal processing, we sometimes filter some prominent frequency components to omit other unwanted and irrelevant components, and this is very similar to what we proposed in that section.

Considering the SVM classification and comparing the results presented in Tables 4 and 5, the benefit of using the diseased hippocampi in the training phase is confirmed. Even after leaving 10 samples out, the accuracy of the trained system for lateralization outperforms the clustering result by almost $12 \%$.

To compare our lateralization results to others, the only available studies using shape analysis for lateralization are [27, 28]. In both of these references, a common dataset consisting of 23 healthy subjects and 31 epileptic patients is used. Patients are with epileptic focus of either Left or Right Anterior Temporal Lobe (LATL or RATL), and from this point of view, their dataset is more specific than ours with patients from different types of mTLE. Their best result of $90.32 \%$ for classification of LATL and RATL is validated by the leave-one-out method. The same cross-validation in our method produced $92 \%$ accuracy. Of course, differences in the datasets can contribute to the differences of the results. However, the power of SPHARM for hippocampus shape classification in MTLE is confirmed.

We can also compare our results to those in [34]. In that paper, mean and standard deviation of FLAIR signal intensities are used for mTLE lateralization. 36 patients (from the same dataset we utilized in this paper) were analyzed and the lateralization accuracy of $98 \%$ was achieved. For this group of patients, the lateralization accuracy by the hippocampal volumetry is reported to be $83 \%$.

To sum up, considering our results and those in [27-29], importance of shape analysis in epilepsy is evident. Our proposed novel LIs can form a system for diagnosis and prognosis of mTLE patients. It can also compliment other methods (e.g. [34]) to decrease lateralization error and increase the likelihood of correct outcome prediction.

For the future work, localized features such as wavelets [16] and Point Distribution Models [12] can be used. We will also aim at using statistical analysis (like SPHARM-PDM in [35]) of mTLE HCs to find areas of the hippocampi that are mostly deformed in our dataset and combine it with localized features to improve the lateralization accuracy further.

\section{REFERENCES}

[1] Csernansky, J. G., Joshi, S., Wang, L., Haller, J., Gado, M., Miller, J., Grenander, U., and Miller, M., "Hippocampal morphometry in schizophrenia via high dimensional brain mapping," Proc. Natl. Acad. Sci. USA 95(19), 11406-11411 (1998).

[2] Convit, A., De Leon, M. J., Tarshish, C., De Santi, S., Tsui, W., Rusinek, H., and George, A., "Specific hippocampal volume reductions in individuals at risk for Alzheimer's disease," Neurobiol. Aging 18(2), 131-138 (1997).

[3] Oppenheim, C., Dormont, D., Biondi, A., Lehéricy, S., Hasboun, D., Clémenceau, S., Baulac, M., Marsault, C., "Loss of digitations of the hippocampal head on high-resolution fast spin-echo MR: a sign of mesial temporal sclerosis," AJNR 19(3), 457-463 (1998).

[4] Duvernoy, H. M., [The Human Hippocampus], Springer-Verlag, Berlin Heidelberg, Germany (2005).

[5] Engel, J., and Pedley, T. A., [Epilepsy: A Comprehensive Textbook], Lippincott Williams \& Wilkins, Philadelphia, PA 19106 USA, chapter 13, (2008).

[6] Dam, A. M., "Epilepsy and neuron loss in the hippocampus," Epilepsia 21(6), 617-629 (1980).

[7] Anstey, K. J., and Maller, J. J., "The role of volumetric MRI in understanding mild cognitive impairment and similar classifications," Aging \& Mental Health 7(4), 238-250 (2003).

[8] Joshi, S., Miller, M, and Grenander, U., "On the geometry and shape of brain sub-manifolds," Pat. Rec. Art. Intel. 11(8), 1317-1343 (1997).

[9] Csernansky, J. G., Wang, L., Jones, D. J., Rastogi-Cruz, D., Posener, J. A. Heydebrand, G., Miller, J. P., Miller, J. P., and Miller, M. I., "Hippocampal deformities in schizophrenia characterized by high dimensional brain mapping," Am. J. Psychiatry 159(12), 2000-2006 (2002).

[10] Bookstein, F. L., "Shape and the information in medical images: A decade of the morphometric synthesis," Comp. Vision and Image Under. 66(2), 97-118 (1997).

[11] Dryden, I. L., and Mardia, K. V., "Multivariate shape analysis," Sankhya, The Indian Journal of Statistics 55, 460480 (1993). 
[12] Cootes, T., Taylor, C. J., Cooper, D. H., and Graham, J., "Active shape models - their training and application," Comp. Vis. Image Under. 61(1), 38-59 (1995).

[13] Styner, M., Gerig, G., Lieberman, J., Jones, D., and Weinberger, D., "Statistical shape analysis of neuroanatomical structures based on medial models," Medical Image Analysis 7(3), 207-220 (2003).

[14] Golland, P., Grimson, W. E. L., and Kikinis, R., "Statistical shape analysis using fixed topology skeletons: Corpus callosum study," Proc. IPMI, 382-388 (1999).

[15] Brechbühler, C., Gerig, G., Kübler, O., "Parameterization of closed surfaces for 3-D shape description," Comp. Vis. Graph. Image Process. 61(2), 154-170 (1995).

[16] Schröder, P., and Sweldens, W., "Spherical wavelets: Efficiently representing functions on the sphere," Proc. Computer Graphics (SIGGRAPH), 161-172 (1995).

[17] Shi, Y., Lai, R., Kern, K., Sicotte, N., Dinov, I., Toga, A. W., "Harmonic surface mapping with Laplace-Beltrami eigenmaps," Proc. of the MICCAI 11th(Pt 2), 147-154 (2008).

[18] Styner, M., Lieberman, J. A., Pantazis, D., and Gerig, G., "Boundary and medial shape analysis of the hippocampus in schizophrenia," Med. Image Anal. 8(3), 197-203 (2004).

[19] Zhao, Z., Taylor, W. D., Styner, M., Steffens, D. C., Krishnan, K. R. R., and MacFall, J. R., "Hippocampus shape analysis and late-life depression," PLos ONE 3(3), e1837 (2008).

[20] McKeown, M. J., Uthama, A., Abugharbieh, R., Palmer, S., Lewis, M., and Huang, X., "Shape (but not volume) changes in the thalami in Parkinson disease," BMC Neurology 8:8 (2008).

[21] Shen, L., Ford, J., Makedon, F., and Saykin, A., "A surface-based approach for classification of 3D neuroanatomic structures," Intelligent Data Analysis 8(6), 519-542 (2004).

[22] Shen, L., Saykin, A. J., Chung, M. K., and Huang, H., "Morphometric analysis of hippocampal shape in mild cognitive impairment: an imaging genetics study,” in IEEE 7th Int. Sym. on Bioinformatics and BioEngineering, 211-217 (2007).

[23] Shen, L., Farid, H., and McPeek, M. A., "Modeling 3-dimensional morphological structures using spherical harmonics." Evolution 63(4), 1003-1016 (2009).

[24] Gerardin, E., Chételat, G., Chupin, M., Cuingnet, R., Desgranges, B., Kim, H. S., Niethammer, M., Dubois, B., Lehéricy, S., Garnero, L., Eustache, F., Colliot, O., and the Alzheimer's Disease Neuroimaging Initiative, "Multidimensional classification of hippocampal shape features discriminates Alzheimer's disease and mild cognitive impairment from normal aging," NeuroImage 47(4), 1476-1486 (2009).

[25] Hogan, E. R., Wang, L., Bertrand, M. E., Willmore, L. J., Bucholz, R. D., Nassif, A. S., and Csernansky, J. G., "Predictive value of hippocampal MR imaging-based high-dimensional mapping in mesial temporal epilepsy: Preliminary findings," AJNR 27(10), 2149-2154 (2006).

[26] Das, S. R., Mechanic-Hamilton, D., Korczykowski, M., Pluta, J., Glynn, S., Avants, B. B., Detre, J. A., and Yushkevich, P. A., "Structure specific analysis of the hippocampus in temporal lobe epilepsy," Hippocampus 19(6), 517-525 (2009).

[27] Kodipaka, S., Vemuri, B. C., Rangarajan, A., Leonard, C. M., Schmallfuss, I., and Eisenschenk, S., "Kernel Fisher discriminant for shape-based classification in epilepsy," Med. Image Anal. 11(1), 79-90 (2007).

[28] Lord, N. A., Ho, J., Vemuri, B. C., and Eisenschenk, S., "Simultaneous registration and parcellation of bilateral hippocampal surface pairs for local asymmetry quantification," IEEE Trans. Med. Imaging 26(4), 471-478 (2007).

[29] Shishegar, R., Soltanian-Zadeh, H., and Moghadasi, S. R., "Hippocampal shape analysis in epilepsy using LaplaceBeltrami spectrum," Proc. of 19th Iranian Conf. on Electrical Eng. (ICEE), 1-5 (2011).

[30] Esmaeil-Zadeh, M., Soltanian-Zadeh, H., Jafari-Khouzani, K., "SPHARM-based shape analysis of hippocampus for lateralization in mesial temporal lobe epilepsy," Proc. of 18th Iranian Conf. on Electrical Eng. (ICEE), 39-44 (2010).

[31] Jafari-Khouzani, K., Elisevich, K., Patel, S., and Soltanian-Zadeh, H., "Dataset of magnetic resonance images of nonepileptic subjects and temporal lobe epilepsy patients for validation of hippocampal segmentation techniques," Neuroinformatics 9(4), 335-346 (2011).

[32] Burel, G., and Hénocq, H., "Three-dimensional invariants and their application to object recognition," Sig. Process. 45(1), 1-22 (1995).

[33] Theodoridis, S., and Koutroumbas, K., [Pattern Recognition], Academic Press, Inc., Orlando, FL, USA (2006).

[34] Jafari-Khouzani, K., Elisevich, K., Patel, S., Smith, B., and Soltanian-Zadeh, H., "Flair signal and texture analysis for lateralizing mesial temporal lobe epilepsy," NeuroImage 49(2), 1559-1571 (2010).

[35] Styner, M., Oguz, I., Xu, S., Brechbuehler, C., Pantazis, D., Levitt, J. J., Shenton, M. E., Gerig, G., "Framework for the statistical shape analysis of brain structures using SPHARM-PDM,” Insight J 1071, 242-250 (2006). 